\title{
Study on the Application of the Heavy Fourier Series with Additional Items on the Bending Problem of Plates
}

\author{
Xianglan Qiu \\ School of Engineering \& Management, Pingxiang University, Pingxiang Jiangxi, 337000, China
}

Keywords: Plate Bending Problem, Fourier Series, Application Study

\begin{abstract}
In this paper, the general bending problem of medium-thickness circular plates on isotropic elastic half-space foundation is systematically analyzed. The deflection of the plate, the load, the ground reaction force and the settlement of the foundation under the slab are all expanded into a double Fourier-Bessel series. The undetermined coefficients in these series are determined by the boundary conditions of the plate, the governing equation of the plate and the plate-foundation. The compatibility conditions are determined to transform the interaction problem between the elastic half-space foundation and the medium-thickness circular plate into the solution of numerical integral and algebraic equations. Numerical calculations show that the series solution has a faster convergence rate.
\end{abstract}

\section{Introduction}

The elastic substrate is a common structural form in engineering, and it is of great significance to accurately analyze it. Currently used ground modes are Winkler mode, two-parameter mode and elastic half-space mode. Although the analysis according to the first two ground models is relatively simple, the results obtained are quite different from the actual situation. Relatively speaking, the elastic half-space model foundation is close to the actual situation. Therefore, the interaction between the elastic half-space and the foundation static and dynamic is an important research topic. Because the elastic half-space model foundation is not only unknown in magnitude, but its distribution law is unknown, accurate analysis of the foundation in elastic half space is a very complicated problem. The predecessors used the power series method (expanding each physical quantity into a power series) to analyze the axisymmetric problem of beams, rectangular thin plates or circular plates on elastic half-space foundation, or use conjugate The integral equation is used to analyze the axisymmetric problem of thin circular plates on elastic half-space foundation, and the analysis process is extremely complicated. Based on the double Fourier-Bessel series, the general bending problem of medium-thick circular plates on isotropic elastic half-space foundation is analyzed by analytical method, and the original problem is transformed into numerical integral and algebraic equations.

\section{Elastic half space solution}

The displacement and stress components of the elastic half space under the cylindrical coordinate system $(\mathrm{r}, \theta, \mathrm{z})$ are $[\mathrm{w}, \sigma \mathrm{z}, \tau \mathrm{rz}]=\sum \infty \mathrm{n}=0[\mathrm{wn}, \sigma \mathrm{zn}, \tau \mathrm{rzn}] \cos n \theta \tau \theta \mathrm{z}=\sum \infty \mathrm{n}=1 \tau \theta \mathrm{zn} \operatorname{sinn} \theta(1)$ and introduce the following stress combinations:

$\mathrm{T} 1 \mathrm{n}=1+\mu \mathrm{sE} \mathrm{s}(\tau \theta \mathrm{zn}+\tau \mathrm{rzn}), \mathrm{T} 2 \mathrm{n}=1 \mathrm{E}+\mathrm{s} \mu \mathrm{s}(\tau \theta \mathrm{zn}-\tau \mathrm{rzn})$

Let $\mathrm{w} \sim \mathrm{n}, \sigma \mathrm{zn}$ be $\mathrm{w} \mathrm{n}, \sigma \mathrm{zn}$ for n-order Hankel transform of $\mathrm{r}$, $\mathrm{T} 1 \mathrm{n}$ be $\mathrm{T}$ 1n for $\mathrm{n}+1$ order Hankel transform of $\mathrm{r}, \mathrm{T} 2 \mathrm{n}$ for $\mathrm{T}$ 2n for $\mathrm{r} \mathrm{n}-1$ order Hankel transform, know

Wn $(\mathrm{k}, \mathrm{z})=(1-2 \mu \mathrm{s}) \mathrm{d} 2$ Yndz $2-2(1-\mu \mathrm{s}) \mathrm{k} 2 \mathrm{Yn}$

$\Sigma \mathrm{zn}(\mathrm{k}, \mathrm{z})=\mathrm{E} \mathrm{s} 1+\mu \mathrm{s}(1-\mu \mathrm{s}) \mathrm{dd} 3 \mathrm{zY} 3 \mathrm{n}-2(1-\mu \mathrm{s}) \mathrm{k} 2 \mathrm{ddYzn}$

$\mathrm{T} \ln (\mathrm{k}, \mathrm{z})=\mathrm{k} \mu \mathrm{s} \mathrm{d} 2 \mathrm{Yndz} 2+(1-\mu \mathrm{s}) \mathrm{k} 2 \mathrm{Yn}+\mathrm{ddXzn}$

$\mathrm{T} 2 \mathrm{n}(\mathrm{k}, \mathrm{z})=\mathrm{k} \mu \mathrm{s} \mathrm{d} 2 \mathrm{Yndz} 2+(1-\mu \mathrm{s}) \mathrm{k} 2 \mathrm{Yn}-\mathrm{ddXzn}$

$\mathrm{Yn}(\mathrm{k}, \mathrm{z})=(\mathrm{C}$ 1n $+\mathrm{C} 2 \mathrm{nz}) \exp (-\mathrm{kz})$

$\mathrm{Xn}(\mathrm{k}, \mathrm{z})=\mathrm{C}$ 3n $\exp (-\mathrm{kz})$ 
Where $\mathrm{E} s$ and $\mu \mathrm{s}$ are the elastic modulus and Poisson ratio of the elastic half space, $\mathrm{k}$ is the Hankel transformation parameter, and C $1 n$, C $2 n$ and C $3 n$ are the undetermined constants. When a normal pressure $\mathrm{p}=\sum \infty \mathrm{n}=0 \mathrm{p} \mathrm{n} \cos n \theta$ acts on a circular area (radius a) on the surface of the elastic half space, the boundary condition of the surface is:

$\Sigma \mathrm{zn} \mathrm{z}=0=-\mathrm{p} \mathrm{n}, \mathrm{T} 1 \mathrm{n} \mathrm{z}=0=0, \mathrm{~T} 2 \mathrm{n} \mathrm{z}=0=0$

\section{Series multi-scale solution of elastic bending problem of thick plate on two-parameter foundation}

As heavy vehicles and large aircraft have begun to become popular transportation tools, especially the rapid development of aerospace technology, airport runways, vehicle yards, and aerospace launching platforms have been continuously thickened, such as heavy aircraft runways and container transport vehicles. The thickness is above the above. In the design and construction of cement concrete floor, the traditional elastic foundation analysis method is still used for structural calculation, and the method of increasing the thickness of the panel is used to further ensure the service life of the project, which may cause huge waste. If the thickness of the panel is too thin, it will lead to a significant shortening of the service life of the field, and even lead to major accidents, delays in large-scale launch missions or traffic jams, and economic and political impacts are great. Therefore, large-scale engineering problems such as launching field lands, airport runways, port yards, and container yards need to be analyzed and calculated using the theory of thick plates on elastic foundations. However, due to the complexity of the differential governing equations and boundary conditions in the thick plate theory, there are great difficulties in both mechanical and mathematical processing, even for numerical calculation methods, such as finite difference methods and finite element methods. ', meshless method, numerical manifold method, finite element line method, basic solution method, abbreviated as, etc., as well.

As a general analytical method for solving differential equations, the series method not only has great theoretical significance, but also shows important practical value in solving the problem of thick plate bending on elastic foundation. For example, under the premise that the bending normal stress is linearly distributed along the thickness direction of the rectangular plate, the linear elastic behavior of the thick plate on the foundation is characterized as a system of equations consisting of partial-order partial differential equations, and obtained by the series method of general analytical form. The corresponding series solution form. When the thick plate is a four-sided free boundary, the convergence of the series solution and the calculation accuracy and calculation efficiency are compared with a variety of load forms. The analysis results show that the series solution has good convergence, high calculation accuracy and can satisfy the load. Except for the vicinity of the boundary condition corner point, and has higher solution efficiency than the finite difference method and the finite element method. In the year, Shi Xiaoping used the superposition method based on the Lie series to deal with the bending problem of the four-sided free rectangular thick plate on the elastic foundation. The elastic foundation still adopts the model, and the thick plate adopts the model. To this end, Shi Xiaoping proposed creatively the concept of transverse shear force, torque, and mean angle of the mid-surface normal around the boundary tangent. The problem of the original solution and its solution is expressed as four-sided slip. The boundary boundary, the thick plate bending sub-problem and its solution to bear the lateral load, and the linear combination of the thick plate bending sub-problem and its solution for the boundary of the sliding branch, the bending moment of the opposite side and the lateral load. The related calculation examples show that the superposition solution satisfies the boundary condition of four sides freely, and has good calculation accuracy. The size of the matrix of the undetermined coefficient formed is the corresponding matrix size in the research work, which further improves the solution efficiency. The research results have been applied to the analysis of the stress of the container yard, and the rational optimization of the pavement structure has been realized, saving the construction cost of a single yard more than 10,000 yuan. In the year, Sun Weiming and Yang Guangsong used the series method of general analytical form to solve the elastic bending problem of thick plates on two-parameter foundation. The general analytical solution established has a good convergence speed, which not 
only has good solution accuracy for displacement and rotation angle, but also for bending. High-order moments such as moment, torque, and shear force also have good solution accuracy, and can well satisfy the four-sided fixed or four-sided free boundary conditions. Using the general analytical solution to calculate and analyze the actual loading situation of the field, some useful conclusions are drawn. For the field slab, the influence of the foundation parameters on the displacement and the internal force is small. When the material constant of the field increases, the displacement decreases and tends to be stable. When the maximum tensile stress increases, the displacement and the maximum tensile stress decrease. Small. These conclusions contribute to the design, construction and maintenance of various large ground surface lands. However, it should be pointed out that the series-based superposition method and the general analytical form of the series method have inherent limitations. Accordingly, the current series of analytical solutions for the thick plate bending problem on elastic foundation can only be combined with specific The load form, boundary conditions, and working parameter combinations are expanded. Therefore, if a new series of method-level multi-scale methods can be introduced in the research work in time, I believe that the situation will be greatly improved.

Since the mid-century, light weight, low rigidity, flexibility, and large-scale have become an important trend in various spatial structures. Undoubtedly lightweight structures can increase the weight of the payload and improve the efficiency of the vehicle. Large structures can expand the function of the space structure. But this also brings a series of new problems to the design, manufacture and use of space structures, in which the vibration control of large space structures has been placed in a prominent position. As a result, the traveling wave active control technology has been quickly recognized and developed as a new structural control method. It is completely different from the classical modal control, that is, based on the modal analysis idea and adopting the modern control theory. The basis of traveling wave control is the traveling wave solution analysis of the wave equation, that is, the motion of the continuum is described by the traveling wave, and the control is controlled. The design criteria of the device is to reduce the amplitude of the traveling wave or to absorb as much of the energy carried by the traveling wave carrier as possible. Correspondingly, traveling wave control technology has two distinct advantages. First, the design of the control law of basic continuums such as one-dimensional continuum strings, rods, beams, etc., which can be described by wave equations, is independent of the boundary conditions, thus decomposing large complex structures. It is formed into several basic continuums, and the boundary conditions and the connection relationship between the continuums are not considered in the first stage of the control design. This makes the traveling wave control method particularly suitable for active vibration control of large space structures. In addition, by using the traveling wave control method, it is not necessary to discretize the structure, which avoids the high-order modal leakage of the control precision in the classical modal control. This advantage is particularly valuable for a large-scale spatial structure that requires a large number of elastic modes to be controlled.

The structure of the traveling wave active control technology has been around since the time it was proposed. Although many achievements have been made in the meantime, the focus of the research is still on the theoretical issues of control law design, stability analysis and measurement method research of basic continuum. In contrast, the wave structure analysis of the underlying waveguide structure is somewhat arbitrary and too simple. For example, when investigating the mismatch control of multiple types of traveling waves in a beam, all researchers are premised on the simple decomposition and independent control of wave motion, without taking into account the cohesive effect between the modes, resulting in actual Control overflow during operation. It can be seen that accurately determining the guided waveguide field in the waveguide structure is of great significance for the effective control of the waveguide structure fluctuation, which has attracted the attention of some researchers.

\section{Conclusion}

In this paper, the Fourier-Bessel series is used to systematically analyze the general bending problem of medium-thick circular plates on isotropic elastic half-space foundation. The comparison 
with the existing results calculated by thin plate theory shows that the method is feasible. of. The series solution of this paper transforms the interaction problem between elastic half space and medium-thick circular plate into the solution of numerical integral and algebraic equations, which is simpler and more general than other methods. Since the selected Fourier-Bessel series is complete in the interval [ $0, a]$, the error of the result obtained by the method is determined by the number of series terms. When enough steps are taken, accurate results can be obtained. This provides a solid theoretical basis for the design and calculation of basic engineering. Numerical calculations show that the series solution has a faster convergence rate. In addition, the method can be extended to the analysis of the interaction between the elastic half space and other structures. For example, using the Cartesian coordinate solution of the elastic half space problem, and the physical quantities are expanded into Fourier series, the elasticity can be solved according to the same idea. The interaction between the half space and the beam and the rectangular plate.

\section{Acknowledgements}

Topic: Application of the Heavy Fourier Series of Supplementary Items in the Science and Technology Project of Jiangxi Provincial Department of Education in the Bending Problem of Plates No.: GJJ161253

\section{References}

[1] Wang Minggui, Huang Yi. Rectangular plates on elastic half-space foundations [J]. Journal of Applied Mechanics, 2004, 11(4): 120-126.

[2] Xia Yongxu. Exact solution of thick circular plate on homogeneous elastic half-space foundation [J]. Engineering Mechanics, 2010, 7(2): 83-92.

[3] Li Gang, Wang Wei, Shang Shouping. Stability transformation of steady-state vibration of rectangular foundation on elastic half space [J]. Journal of Hunan University (Natural Science), 2000, 27(4):88-93.

[4] Liu Pengcheng, Yang Wenbo, Li Chang. Curved curved multi-fork tree model and its application in map synthesis [J]. Computer Application Research, 2011, 29(7): 2793-2795

[5] Ai Tinghua. Granularity control and sequential control in network map progressive transmission [J]. China Image Graphics, 2009, 14(6): 990-1006 